\title{
Spaces, Semantics, and 'Aesthetic Faculty': Perspectives on Their Operative Plane of Concurrence, Constitution and Becoming
}

\section{Dhanesh M.}

\author{
Assistant Professor \\ English and Cultural Studies Department \\ Christ University \\ Bangalore, India \\ dhanesh.mankulam@gmail.com
}

\begin{abstract}
This paper aims to look at one of the fundamental factors of human beings - the appreciation of things. Calling it 'the aesthetic faculty' this paper tries to see how it is inevitable to the way human beings as a species function. This paper aims to propose this idea of an 'aesthetic faculty' as a potential basis for our community life in its diverse operations in terms of cultural spaces and their semantics. Viewing the socio-systemic life from the point of view from the aesthetic faculty reveals how appreciation and evaluation are inevitable to human life and how an ideological ground cannot actually affect life without addressing this basic human faculty. This paper tries to take the term 'aesthetic' vis-a-vis 'appreciation' to a different semantic world altogether so that it is no longer a matter of artistic engagements alone, but something more fundamental and formative than that.
\end{abstract}

Keywords: Spaces, Semantics, Aesthetic Faculty, Appreciation

It is quite slippery when we talk about culture, life and an ideological context in the contemporary discursivities when we actually find less efforts in a direction through which 
we may arrive at a factor more fundamental to the whole paradigm of politico-cultural studies than anything else - the factor pertaining to the human faculty of appreciation. An immediate rational reduction might make this idea of appreciation appear simple and too obvious to be of any critical contemplation. A serious thought, on the other hand, reveals that this tendency to appreciate and evaluate things is constitutive of our lives; overtly as an experience and covertly as a binding force that in fact unite the whole ensemble of social relations and semantic systems. I will argue in this paper that human tendency for appreciation shall be considered to be a basic inherent faculty — may it be called 'aesthetic faculty' (but with a whole lot of fresh semantic underpinnings to the already familiar term) — and it acts not only as the binding force behind what we may call 'lived-life' but also as a ground for all the semantic, hierarchized and functional derivatives of that socio-systemic ensemble including politico-cultural spaces. This paper aims to propose this idea of an 'aesthetic faculty' as a potential basis for our community life in its diverse operations in terms of cultural spaces and their semantics. Viewing the socio-systemic life from the point of view from the aesthetic faculty reveals how appreciation and evaluation are inevitable to human life and how an ideological ground cannot actually affect life without addressing this basic human faculty. I must disaffiliate the term 'aesthetic faculty' from the commonly used sense of the term 'aesthetic'. The traditional schools of Aesthetics considered aesthetic to be a philosophy of art and as an appreciation of beauty. As Kant discussed in the Critique of Judgement the attempt was to analyse certain state of mind involved in the very faculty of appreciation of beauty. Edmund Burke, on the other hand, aimed at the language and concepts involved in the aesthetic criticism. If Immanuel Kant, David Hume and others pioneered the discourse in the earlier times, recent times have seen a variety of discursive vectors developing in close correspondence with the existing discourses on culture, Dalit and minority experiences, gender and subjectivity, and socio-political dimensions including Late Capitalism, New 
Leftism, Everyday Life, fashion and so on. However, it has been considered to be a matter of appreciation of beauty and taste in art and elsewhere. This idea of appreciation when considered against the backdrop of the present politico-cultural studies reveals something that is more fundamental to the latter. This paper develops through an analysis of the creation of sense and space, two central notions in contemporary Cultural Studies.

The paradigm set by modern Cultural Studies and similar discourses on culture seems to have a different approach — one which is rather less focused on the idea of aesthetics as a basic faculty of appreciation. The term 'faculty' here hints at the idea of appreciation as an inherent quality or characteristic of human beings. Cultural studies, whether it be the foundational theoretical developments from the West, or it be the discursive writings within the established domain of Cultural Studies worldwide, attempts have been mostly to look at cultural space in terms of the semantic, semiotic, political, empirical, epistemological and even existential productions that happens in and around it. According to Stuart Hall, for example,

At the heart of the meaning process in culture, then, are two related 'systems of representation'. The first enables us to give meaning to the world by constructing a set of correspondences or a chain of equivalences between things - people, objects, events, abstract ideas, etc. - and our system of concepts, our conceptual maps. The second depends on constructing a set of correspondences between our conceptual map and a set of signs, arranged or organized into various languages which stand for or represent those concepts. The relation between 'things', concepts and signs lies at the heart of the production of meaning in language (Representation 19)

These theories tie the concept of cultural space to some kind of a semantic production - as codes, representations, practices and norms. Correspondingly, we are familiar 
now with terms such as sense, sign, symbolic, power/knowledge, life of the everyday, lived life, knowledge, subject-object, ethics, desire and so on. We also hear about terms such as ideology, power, individual, and life more often than not in an entirely novel context of significations and relations. Moreover, dynamic, constitutive, signifying, functional, and becoming have become commonly used adjectives for this field at present. Most of the discussions and discursive activities have been happening around these terms and themes which are more or less based on a larger concept of 'semantic production' or simply 'meaning making'. It is doubtful if adequate attention has been given to arrive at a more fundamental aspect upon which both these discursivities and the corresponding real-life interactions actually function. This is where we can think of aesthetic appreciation as an inherent human faculty. This idea seems to hint at something relevant and more basic to the existing understanding of cultural space and life in it. This anchoring, though not absolute, seems to give Cultural Studies a fresh orientation that aids in its approach and desire to change.

Pramod K. Nayar published an interesting work titled Packaging Life: Cultures of the Everyday in 2009. Using 4 terms - health, comfort, risk and mobilities - he tries to study cultural politics which “... involves the construction of senses and values through a strategic use of representations, narrative and rhetoric. Such representations mask the ideologies behind the senses of products, events and conditions. In other words, products, services and conditions instantiate discourses and, therefore, politics" (emphasis added, vii).The book studies the way in which various aspects of 'everyday life' “...acquire senses such as good health, cosmopolitan identities or luxurious lifestyles (emphasis added, vii). A question immediately strikes here: a question not only about the end of cultural studies as studying the production of senses, but also about those adjectives—good, cosmopolitan, and luxurious. Despite being indicative of quality or degree, those adjectives are terms of comparison too. 
They are the key to how life acquires senses. Moreover, they refer to what one chooses over an array of plausible alternatives thereby making the acquired sense political. In fact, reasoning from Pramod K. Nayar's observation, it is in this 'choosing' that contemporary life gets its credits to be 'packaged' and consumed. What is that factor which influences or determines or even grounds this choosing after all? I would call it 'aesthetic faculty' —an inherent characteristic tendency of human beings which might seem simple but is in fact grounding everything from one's everyday choices to the human model of language.

In that sense, I would suggest that, while agreeing to Pramod K. Nayar's statement that "politics... is essentially about power, ideology and the control over people, ideas and behaviour, where ideology works mainly through suggestion, advice and opinion” (xxi), I would supplement by stating that those factors such as suggestion, advice and opinion operate upon a pre-set conceptual base of 'aesthetic faculty' which is inevitable and fundamental to the human species. This base(of the aesthetic faculty) provides the elementary patterns upon which the dynamics of cultural productions as senses, relations, hierarchies and so on can effectively engage in an indeterminate Derridean play. If someone thinks that this pre-set habitat for cultural productions is set by culture, I will partially agree but with a footnote to it: it is, like sexuality, both cultural and biological at the same time. It has a biological base, but its manifestation involves a cultural mediation; it emanates from our 'instinctual domain', but it takes up cultural forms. Moreover, this is one of the faculties that demarcate humans as a species from other animals.

Pramod K. Nayar devotes one section titled The 'Stylization of Life' Itself to talk about the aesthetic aspect of life. He uses the term 'style' to denote this. As he says, "...utility and functionality of goods must be accompanied by an aesthetic appeal—what I have simply denoted by the term 'style..." (Packaging 54). He defines style in these terms: "style is the uneasy and complex negotiation between determining one's own style and finding acceptance 
among one's peers through a common code" (60). What I find missing is a question on the unavoidability of this stylising as a cultural core. Why do we see this style, though in varying forms with varying meanings, across cultures? That is where an idea of aesthetic faculty comes in: something that has a biological frame yet only manifested in available cultural terms. Rather than a market relation that binds together market goods, consumers and the desire for consumption, I find this to be even more fundamental to the way we operate as intelligent animals. Moreover, this aesthetic trait may be defined not in terms of visual, aural or olfactory styling, but in terms of the faculty of creation and retention of certain patterns of appreciation and evaluation among a multitude of possibilities. It is a tendency to choose that can be seen in every aspect of life. It happens among species members, and beyond. In countries like India this is more evident aligning with discourses on purity and racial lineage. It is not proper ideological adherence that binds people here; rather it is a commonly shared engagement of this aesthetic faculty that binds them. It is uncontested that this shared structure is socio-cultural while the underlying faculty is not absolutely socio-cultural. It is biological as well. That is why we can call it a 'faculty of appreciation'.

How this faculty which is both biological and cultural at the same time can be imagined in terms of other animals? I would say that a kind of choosing works in animals as well. But, like sex or reproduction, this is rather biologically coded and operated than culturally or 'abstractly' mediated. For example, animals choose between eatable and noneatables, but they seem to lack a gradation among eatables especially based on contexts and moods. Hence, I may not call it 'aesthetic faculty' though a similar faculty works in them as well. Here we step on to another nuance of what might be called the aesthetic faculty of human beings: though it is an underlying biological frame, it has to have a culturally mediated structure for its actual manifestation. Think of sexuality: it has a biological drive in 
it but is cultural in its manifestation to the greatest extent. In short, aesthetic faculty is imagined to be a human trait here.

Coming back to the thread, while stating that the "issues of style, fashion, aesthetic appeal are not properties inherent in particular objects, but the result of a social and cultural evaluation of these objects" (Nayar, Packaging, 49), we must also say that this evaluative function, or this act of appreciation is something really fundamental to human life in the sense that it makes happen everything else as its derivatives. Even when the actual 'choosing' that we make is cultural and systemic, and can vary from contexts to contexts, the 'faculty of choosing', like a general linguistic structure with a fundamental I-vs-Other structure, seems more essential and even inherent to human life as a species. Other factors such as quality, utility, success and sense are derivatives of this fundamental tendency. It would be somewhat naive to refer to an example, an empirical evidence, to designate this factor in relation to life though, for the faculty [as a potential] has an empirical dimension only in its manifestations which are cultural. But it is indispensably part of the totality of the system of life that humans as a species wield. In other words, like language, aesthetic faculty adjectivizes systemised human life in general and as a whole. This also implies that this aesthetic faculty is indispensable and should primarily be addressed in order for any ideological current to actually have some effects on human life.

The lack of a clearly stated reference to the correlation between the aforementioned aesthetic faculty and the socio-political and cultural systems of functions, relations, productions and consumption seems evident across existing theories on culture and life. While Cultural Studies as a domain of critical enquiry does address both individual and collective, the focus seems to be more on what the individual experiences as part of various collective cultural phenomena. This argument is not against the general distrust for a metadiscourse — the 'incredulity' towards all meta-narratives that Lyotard noted in his 
Postmodern Condition — that imagines a standpoint outside the 'play' of discursive elements; rather it seems that we can still validly link the various micro discursivities with a more common basis, here as an inherent faculty, just as we do with our model of language. The same happens to our study on power, politics, and Capitalism as well: we often miss the general for the particular.(For example, we miss out a lot when we do not count the global context of Capitalism in our approach to the regional or local instances). The lack that I pointed out in the contemporary academia in addressing what I call the 'aesthetic faculty' seems related to the general distrust for the common and collective that occurred at the onset of post-structuralism. We can recognise that lack as we move, say, from Adorno to the present time.

The question that comes up immediately must be concerning the function and effect of this basic aesthetic faculty. (As noted already, we must take this as a biological frame manifested in cultural forms). Straightaway one can state that the primary function of this aesthetic faculty is that it creates an imaginary sense of quality based on which differentiation becomes possible. It enables us to qualitatively measure the otherwise unqualified life. (One can recognize a possible detour to Biopolitics at this point. I am not exploring that area for this paper though). In other words, it adds a semantic tag to every aspect of life thereby making the various aspects of life to come to a virtual existence in the orders of signification, senses and qualities. Putting it differently, aesthetic faculty is at the base of all the productions and relations as a primary condition. Production of senses or meanings is a recurring point in Cultural Studies. Envisaging aesthetic faculty as the basis of these productions seems to supplement the priority given to the 'product'-here meaning or senses - with a background of a 'production ground'.

What is the nature of the senses produced? And what about cultural spaces within which senses function? Cultural Studies tells us that senses are contextual, produced in 
certain cultural spaces. But senses that are produced in the cultural spaces through various exchanges and interactions are always qualified and differentiated among themselves.

Notwithstanding the relation of these senses with the individuals and objects involved in cultural exchanges, these senses position themselves in relation to one another in the cluster of different senses and their varying shades. We can even use the expression 'qualified senses', as these senses(as a cluster of senses) essentially qualify the various aspects of life. Moreover, they qualify each other by being in a relational position among themselves in the cluster of senses. Most of these ideas are existing ones while I am specifically pointing at a qualifying cross-reference that exists among various shades of senses, thereby animating the entire system of signification. This is a somewhat linguistically institutionalised territory of inter-references.

This cross-reference among senses constitutes a semantic habitat to every [shade of] sense or meaning that is generated.The actual [functional]sense depends upon the semantic habitat (along with various other factors) within which every sense is qualified and differentiated by innumerable cross references among multiple senses. In other words, this is the characteristic feature of the signifying system that we live in. What we can designate as 'actual sense' is that shade of sense which wields a functionally significant affinity to the general normative functions and exchanges of the particular cultural space. Obviously, this qualification is not neutral: it effectuates a gradation of meanings among themselves, which in turn qualifies and grades all the otherwise assorted dimensions of life. Location of a sense in this semantic habitat determines its actual agency as a semantic element with its social status and accessibility. Sense, like individual, has a definite social status, accessibility and agency in its respective cultural space. Now, the question is about the nature and effect of this graded qualification and the sense or meaning thereby produced. 
In the field of contemporary Cultural Studies, we often come across the argument that meaning is what a particular cultural space generates. The SAGE Dictionary of Cultural Studies notes in its entry on the 'circuit of culture' that ... cultural meaning is produced and embedded at each level of the circuit that is, production-representation-identity-consumption-regulation, so that the production of significance at each moment of the circuit is articulated to the next moment without determining what meanings will be taken up or produced at that level (Barker 22).

Pramod K. Nair sets his objective of his book to see how "aspects of everyday ....acquire meanings" (Packaging vii). This kind of reference to 'meaning' or sense is commonly seen in Cultural Studies. Even an argument that sense is the end-result of cultural production stands for a genuine contemplation. In other words, there is a significant assumption that cultural space is the functional and graded ensemble of senses. One imbalance that comes with this assumption is that it more or less implies a precedence and prioritization of the idea of a cultural space over the various senses that comes up in it. This assumption seems rather questionable. What imagination of space can there be if it is not essentially pertaining to some graded senses? Do we have something like an absolute space before it begins to mean something? Where should we draw a demarcation in between the production of space and sense in the pragmatics of life? My response to these questions will be to indicate a reasonable simultaneity in the emergence of cultural space and sense.

Space is what is born with the production of certain graded senses. Hence, there is no singular independent referent as 'space' within which certain semantic or cultural productions take place. There is no 'entity-space'. Instead, just like sense that is born in each event of cultural exchange, cultural space is produced simultaneously with the production of senses. Perpetuation of a seemingly singular cultural space and sense implies a series of 
reinstatement of the same which underlies the signifying system. Shall we draw an analogy with Judith Butler's point on how gender is constituted in each performative gender-act? We may do so. Butler notes in Gender Trouble that"... performativity is not a singular act, but a repetition and a ritual, which achieves its effects through its naturalization in the context of a body, understood, in part, as a culturally sustained temporal duration" (Butler xv). This seems to apply to the 'space-sense essence' in the cultural domain as well. Similar to gender essence created in gender performativity, the seemingly existing 'space-sense essence' is an anticipation within a ritualised domain of space-sense production. In the present discussion It tries to see space as being created instantaneously and simultaneously in and with the production of certain senses. The nature of the production of sense and space is, to appropriate Deleuze's notion, that of becoming.

This concept of cultural space implies that it is dynamic and relational. Space is produced in each instance of sense-making. Space is an event which has a chance 'not to be what already exists' as much as to be a perpetuation of what exists. A conceptual link can be made to Foucault's 'power-resistance' model, where power and resistance are potentials generated simultaneously at the relational knots of social exchanges. In similar terms we say that not only sense but also space is generated instance-to-instance. Moreover, space changes in accordance with the position that corresponding sense occupies in the cloud of innumerable senses: to put it straight, space is created instantaneously with each fluctuation in the cross references in the semantic habitats of senses. The fact that there are different senses creating a habitat of senses within which everything gets semantically qualified and even validated underlines another fact that there is a 'semantic hierarchy'- the order in which particular senses get different shades of qualifications in the respective habitat of senses. This must, by logic, influence the hierarchical nature of cultural space as well. 
It is a fact that human beings basically operate on a linguistic model. Cultural Studies has made us aware of how meaning is not just a linguistic production, and how various other socio-political factors become central to the production of a sense. As has been discussed, the sense of an utterance/exchange is always caught up in a cloud of senses in which each of the shades is differentially positioned on an order of appreciation ranging from worst to best. Implied meanings can be cited as an example of this differential qualification. We have abusive words in language because of this differential qualification. This enables individuals to use linguistic utterance on an evaluative scale between worst and best. This involves both linguistic and extralinguistic aspects. The aforesaid habitat of cross-references functions within the linguistic dimension. Other factors such as material contexts, economic relations, other relations, situations, tradition, practices, epistemology and power form the extralinguistic dimension. If the semantic habitat effectuates one level of graded qualification, these aspects effectuate another level of graded qualification of senses. This latter must be differentiated from the former. Moreover, qualification in the self-referential semantic habitat functions in a manner similar to the operation of signifiers in the Saussurean model of a signifying system.

This qualification of sense owes much to the basic human tendency to appreciate and evaluate things. Appreciation and evaluation come as a natural cumulative achievement of this two-levelled qualification. Aesthetic faculty shall be the basis of the way we make a selection between the best and the worst. This happens among the senses, among spaces and then the rest of our acts of perception. Various structures of power, knowledge, morals, norms, ethics and codes are the palpable manifestation of the grading function of the aesthetic faculty leading to an evaluation of every empirical data. These structures - of power, knowledge etc. - are derivatives of the fundamental faculty of gradation, appreciation and evaluation, that is the aesthetic faculty. This faculty has a biological dimension to it; 
meaning it is unavoidable and essential to our understanding of the cultural forms which is the other dimension of this faculty.

If the I-vs-Other model of language operates as an inevitable linguistic faculty in human species, the aesthetic faculty complements this linguistic faculty of human species by linking it to our empirical life via a hierarchized semantic system. Ido not intend to say that the latter is separate from the former; rather, it may even be seen as one way in which the whole frame of intelligence functions. The point is that the aesthetic faculty is not a secondary derivative of any more basic linguistic function; rather it is inherent to human beings in general as is the linguistic faculty. Hence, aesthetic faculty is a force that helps the production of sense and space, which in turn is the basis of the many derivative functions of the linguistic model that we practically live in. We may also state at this point that, like difference and deference, function of this aesthetic faculty must be a third property of the whole signifying system and its functions which are specific to human species. That explains the inevitability of appreciation, evaluation, choosing, and sense-making in life. Aesthetic faculty is as inevitable to human species as the binary model of language. In that sense, in the I-vs-Other model of language, both I and Other indicate two directions of engagement with the aesthetic faculty; meaning two directions of the act of appreciation and evaluation. Moreover, the directions are constitutive and reciprocal to each other. This also means that changes in the way we engage this I-vs-Other model with the aesthetic faculty will change the nature and order of the cultural sense and cultural space that such engagements produce.

Talking about this differential qualification of senses, we know that all senses are not equal; they gain different momentum at different spaces.(We should also keep in mind that sense and space are not really separable). That is why an utterance that tries to generate a sense yields different shades of sense (or same meaning but different qualification) in different semantic habitats. The sense thus produced can vary in its position in the semantic 
hierarchy, in between the aesthetic extremes of best and worst, also depending upon the extra-linguistic factors that have been noted earlier. So, even when we state that there is a dual function involved in the production of space and sense, we should also note that they operate cumulatively, as a twofold event, and corresponding spaces emerge simultaneous to the emergence of senses. The various spaces, and 'space-within-space' are created across this semantic hierarchy, and appreciation happens based on the particular sense's position in the hierarchy. Just like the hierarchy among relations of power, this semantic hierarchy also is a dynamic hierarchy in which discourses can influence or even determine the position of senses. One [shade of] sense can undermine another [shade of] sense and come to prominence as the actual sense any time. Something obscene can turn into something best and vice versa with a consequent effect in the respective semantic space. It seems less farfetched to say that these varying senses are not altering the existing spaces, but creating new ones, as if from a Hegelian synthesis. In short, spaces and senses emanate from each cultural interaction. Cultural spaces and senses are produced in the micro-interactions of the everyday.

What makes this hierarchy? The answer shall not lead us to any originary point. This hierarchy is the structure of the whole cultural ensemble of our social life. Just like the question what makes the I-vs-Other structure of language, a theological model of question concerning an absolute origin seems to be leading us nowhere. The roots of this aesthetic faculty vanish into our biology. (We might be able to continue to think along the line of Evolution theories and so on). We should not forget that aesthetic faculty has a cultural or systemic counterpart as well. The semantic hierarchy is the property of this faculty of appreciation that enables us to differentiate and distinguish things into various categories of value. Moreover, this hierarchy makes the whole signifying system political as well as a system of privileges by differentially providing agency and authenticity. Think of this in line 
with Foucault's notion of the relations of power that determines the agency and authenticity in relations. For a better understanding of the point we can relate this hierarchy to the hierarchy of body and sexuality in which human body is sexualised differentially over a semantic order.In terms of sexuality, body parts have different meanings, play different cultural functions, and are different cultural spaces even within what we may call a whole body. This differential sexualising gives the different parts of the body different level of agency and authenticity in sexuality.

I am reminded of one of Deleuze's reference to people's desire for fascism in AntiOedipus. Michel Foucault refers to it in his preface to Anti Oedipus as "...the fascism in us all, in our heads and in our everyday behaviour, the fascism that causes us to love power, to desire the very thing that dominates and exploits us" (Foucault xiii). I agree to the point that there is a desire for a centre in the basic structure of our social life. People desire to have a responsible centre, an accountable authority, that can preside over life in its entirety and variety. As is clear, this centre can be of multiple forms and formulations, such as God in religion, President in State or parties, truth/morality/ethics/justice in ethical-subjective domain of life and so on. It seems reasonable to imagine that this 'desire for the centre' is what makes the species-life of human beings hierarchically structured, differentially mediated, and semantically signified and coded. The desire for appreciation, evaluation, and selection have close links to this desire for a centre. May it be God, leader, heads, meaning or truth, no need to mention more, we have always been with centres. Nevertheless, we have been identifying and deconstructing centres with the dawn of the 'post' era. Still, it is explicit that we are nowhere in being without centres. We can see, especially today, people aspiring, praising or commemorating centres that authoritatively preside over lives. I might go further from this thought to state that this desire for an authority is a basic thought-structure that complementarily work with the aesthetic faculty. 
The 'desire for authoritative centre' has its roots in the aesthetic faculty which, as mentioned, helps one make 'certain qualified sense of things' thereby making an appreciation and evaluation possible. This desire is the one formal outline to the possible manifestations of the aesthetic faculty. Other factors - like appreciation, semantic hierarchy, significations, qualifications, semantic spaces etc. - then fills this formal outline of the desire for centre, and derives an array of derivatives which are rather immediate and sometimes concrete, like religion, state, ethnic groups, language, semantic-semiotic systems, knowledge/science and sexuality, to cite a few. The combined operation of aesthetic faculty, acts of appreciation/evaluation, formation of centres/authorities, creation of semantic habitat and its hierarchies may be plotted on a simple representation like this:

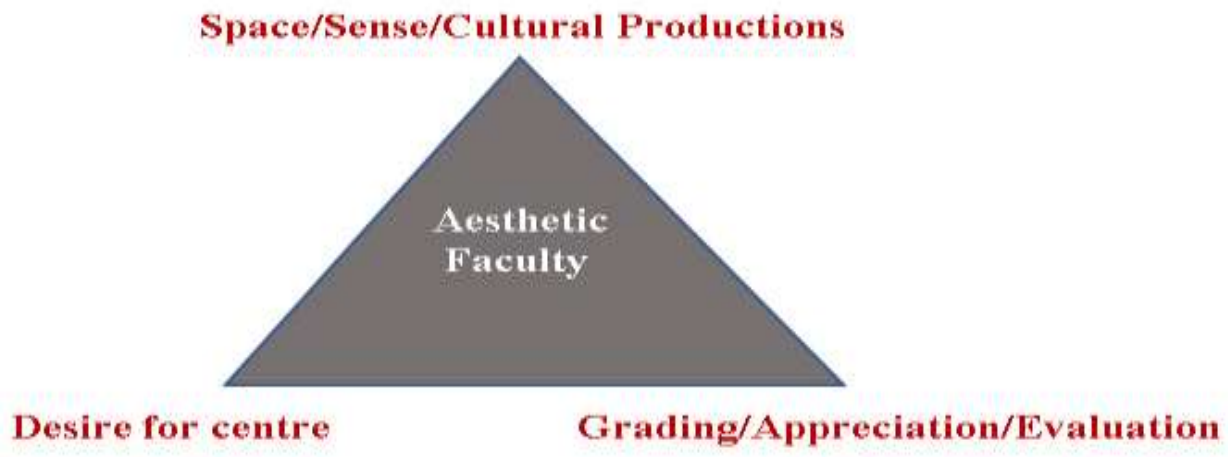

I shall mention two vectors that converge at an individual's subject-position which make the individual subject an ambivalently situated operative point in a process leading to a system of '[aesthetic]evaluation'. The ambivalence comes in in the fact that at this point the individual subject (a member of the social ensemble) becomes a subject and a sovereign at the same time. On the one hand the individual subscribes to various discursive statements of the normative centre — like God, Truth, language, leader etc — to fashion himself/herself as the sovereign centre's 'good subject'. On the other hand, the same subject imagines itself as a potential sovereign capable of evaluating and checking the 'other' in terms of morals and 
norms. It is the submission that enables the subject to be a sovereign in itself. If we mark arrows representing these interests, they will be vector lines moving in opposite directions: one as subject and the other as sovereign. This is important because, it is only by positing oneself as a sovereign or centre that one can engage the aesthetic faculty of evaluation, gradation and hierarchization. This positing is possible only when a sovereign or centre is recognised, and the subject is submitted to that centre. In other words, welcoming an evaluation by the centre enables (or disables) the subject to evaluate the 'other'. This is how asocial establishment, a semantic structure, a hierarchized order is built around the otherwise biologically unqualified life to mediate, evaluate and process it. In a sense, this multifarious movements, based on the aesthetic faculty and its potential manifestations, can be cited as one way by which the biological life is mediated and translated into a socio-political form of life. These multifarious movements and forces, based on the aesthetic faculty, effectuate what we can call in general terms 'establishments'—social, political, cultural and so on.

Thinking about the pragmatic dimension of these discussions, I would like to initiate from are iteration that this aesthetic faculty is not only inevitable and fundamental to human life but also foundational to the structure, strategy and effect of our socio-political and cultural life-fields and its various establishments. It is foundational as a necessity to the semantic and semiotic orders of human life. The kind of establishments that we have in our life is determined primarily by the kind of engagement that we make with our aesthetic faculty and its manifested forms. Hence, any change in an established set of relations and hierarchies can be had only by engaging the aesthetic faculty differently. Moreover, any radical 'demolition' or deconstruction cannot go longer without providing alternative means of engaging the aesthetic faculty just because it, like language, is inevitable to human life as a case of our species life. Any radical ideas intended to subvert or deconstruct the existing structures, hierarchies and their effectual domain must not only be deconstructing the existing 
aesthetic engagement that leads to a particular practice of the appreciation of $I$ and Other, but also be hinting at an alternative way of engaging with the aesthetic faculty thereby suggesting an alternative way of imagining the reciprocal relation between the $I$ and Other. We are still not far from this definition of cultural criticism here:

Cultural criticism... would not only try to raise the reader's suspicion against the supposed false innocence of the forms and transparencies of language that hides the self-interested conventions that tacitly bind together value, meaning, and power. Additionally, it would try to excite the critical imagination concerning the fissures between reality and its others that art suggestively maintains open, so that the reader is motivated to break the mould of refabricated meaning with an unmaking and remaking of a free subjectivity that lets itself be attracted by the categorical unknown and wandering words (Richard Cultural Residues 12)

But the point is that to break the mould, to refabricate the relations and meanings in a new way, the aesthetic faculty must be engaged in a different way. Neglecting this fundamental species-trait, a de-making and re-making may not effectively happen.

It is obvious that aesthetic engagement at the level of social praxis has a collective form to it. There is certain commonality, which we may call norm, practice, ethnicity, culture etc. to which everyone of a community subscribes, and there generates a common standard, a common network, of appreciation and evaluation. The social ensemble and its reality exist only because each member of the society is installed into a network of appreciation characterised by a relation of appreciation. This network has, in Lacan's terminology, the nature of the Symbolic Other. In the Ecrits he often refers to it as symbolic chain, hinting at the nature of the symbolic Other as relational. According to Lacan, it is with reference to this Symbolic Other that every enunciation takes place. It is by sharing the order of the symbolic, 
by being its object in common, that we make not only sensible interactions in our life but also our sense of reality in general. According to Zizek, “...displacement of our most intimate feelings and attitudes onto some figure of the Other is at the very core of Lacan's notion of the big Other. It can affect not only feelings but also beliefs and knowledge - the Other can also believe and know for me" (How to Read27). Thinking about the character of the Symbolic Other we can see that it is primarily based on a relation of appreciation; an appreciation that has been displaced from particular individuals to a network of relations of appreciation. When you dress up in a particular way, when you regulate your postures in a particular way, when you perform your gender in a particular way, you are putting yourself to the appreciation of the Symbolic Other, though with differences depending up on the way you position yourself against the Symbolic Other. Then, you are also positioning yourself in relation to a multitude in the network of appreciation of which every one of the society is an active functional part.

Engagement of the aesthetic faculty as a collective function of evaluation takes place among individuals and within individual in a network of innumerable cross references. The relations in this network themselves constitute the Symbolic Other as the big eye that monitors, sanctions and validates every semantic dimension of human existence. As Zizek notes, the Symbolic Other”... never existed in the first place, i.e., the "big Other's" inexistence is ultimately equivalent to its being the symbolic order, the order of symbolic fictions which operate at a level different from direct material causality" ( "The Big Other"). But we can say that the 'big Other' exists as a network of relations of appreciation in which the engagement of the aesthetic faculty is done collectively to form a standardised pattern of such an engagement. The Symbolic Other is then this network of relations of appreciation to which all individuals are connected as functional knots, receiving and transmitting certain relations of appreciation. This is analogous to Foucault's notion of the network of power 
relations that excludes none, and that generates from all. The open-endedness of the constitution of the subject and the Other is at least partially determined by the open-ended momentariness of the 'appreciation-bracketing' caused by the engagement of the aesthetic faculty. In that sense, engagement of the aesthetic faculty has been there from time immemorial. Complementing Pramod K. Nayar's statement that "consumer culture in the late 20th century is marked by several simultaneous shifts from 1 . use to display 2 . function to artistry 3. utility to aesthetics (Packaging61), we must also remember that this 'aestheticism' has been there in different forms and meanings always as part of our cultural life. If the late Capitalist turn has a much more emphasis on the aesthetic factor as a kind of relationship, that needs to be credited to Capitalism's unparalleled skill for adaptation and appropriation. The fact remains that ideologies, no matter Capitalism or Communism, have been basically engaging and disengaging with this aesthetic faculty differently.

I shall conclude this discussion with one more note. Here I am thinking about the general spread and success of Late Capitalism vis-à-vis the struggles of the Left ideologies, especially in a country like India. I am not going into the statistics to back this assumption, for the point I want to make stands for itself anyway. Any radical critical idea or movement is supposed to address the social-political-cultural establishments generated by the certain patterns of engaging the aesthetic faculty. On the one hand the movement or ideology must deconstruct the existing aesthetic engagement and on the other hand it must make a shift to an alternative engagement of the aesthetic faculty. Even when the ideology and modes of life change, the aesthetic faculty as such, as a biological instinct, remains the same. Only the manifested forms change. My observation is that while both Capitalism and Left ideology can (and do) make paradigm shifts, the Capitalist strategy seems to have an alternative engagement to offer always, whereas the left ideologies in their practical political forms, seem not to have a clear statement about an alternative engagement of the aesthetic faculty. 
Taking Althusser's thesis - that production demands a reproduction of the material conditions of production, which demands the reproduction of the relations of production, and that ISA and RSA secure this chain of reproduction-into consideration one can even state at this point that this reproduction of the relations of productions in the cultural domain (cultural ISA) has at its base a fundamental faculty which secures the entire ensemble of semantic and spatial relations. One can also state that it might be this biological base that necessitates particular formation and sustenance of certain patterns of ideological operations. In the case of Late Capitalism, it succeeds in maintaining the necessary relations of productions across instances of social transformation because by producing an alternative cultural framework of consumption, appreciation and evaluation, it can engage the unavoidable aesthetic faculty. For instance, when you have to shift from a religious orientation, with all its ideas of good and bad, to an urban market orientation, you are lead to a different set of ideas of good and bad so that you still have a semantic habitat intact where you can qualify and evaluate life and the multifarious cultural productions in it. On the other hand, a Left movement, while it leads you out of the existing modes of evaluation of life by problematising them, its offer of an alternate is rather vague or distant. The fact that the former can hold multiple shades of evaluative standards (religious + scientific, segregationist + democratic etc.) at different domains of life without an inner conflict seems to take an advantage here. Taking Simon During's thesis that "in its most radical guise, the neo-Foucauldian thesis argues that culture is neither an end in itself nor the product of autonomous agents - whether individuals or communities — but a mechanism for transmitting forms of 'governmentality', for ordering how we act, think, live" (During Reader 16) one can even see that the aesthetic faculty as a bio-cultural mechanism functions as an agent that structures all forces and relations of governmentality. It is high time we conceptualize a bio-cultural dimension to our studies on culture as well as biopolitics. Aesthetic faculty is one instance to this. 
In short, this aesthetic faculty anchors the cultural centres - centre in the sense of 'binding centre' or the 'centre that holds the multitude'-manifested as different relations of appreciation and evaluation with which the signifying system functions. This function is in terms of the instantaneous and recurring constitution of identities (in a broad sense). The nature of this function is, as established in the existing theories, a kind of Deleuzean becoming. Hence, in our critique of cultural establishments, we must address the fact that this aesthetic faculty, on a common operative plane, constitutes and co-operates with a concurrent and cumulative function of cultural space and sense with a nature characterized by a dimension of becoming. 


\section{Works Cited}

Hall, Stuart,edited. Representation: Cultural Representations and Signifying Practices. The Open U, 1997. <https://pdfs.semanticscholar.org/90bd/e6eeb7933bd20221ec9bda0e51a8d94d40e1.p df.

Foucault, Michel. Preface. Anti-Oedipus. By Gilles Deleuze and Felix Guattari, U of Minnesota P, 1983.

Richard, Nelly. Cultural Residues: Chile in Transition, Translated by Alan West-Durán and Theodore Quester, U of Minnesota P, 2004.

Zizek, Slavoj. “The Big Other Doesn't Exist”, European Journal of Psychoanalysis, Number 5, 1997. <http://www.psychomedia.it/jep/number5/zizek.htm>

---. How to Read Lacan, Norton, 2006.

Barker, Chris. The SAGE Dictionary of Cultural Studies. Sage, 2004.

Butler, Judith. Gender Trouble: Feminism and the Subversion of Identity. Routledge, 1990.

Nayar, Pramod K. Packaging Life: Cultures of the Everyday. Sage, 2009.

During, Simon, edited. The Cultural Studies Reader, Second Edition, Routledge, 1993. 